\title{
Development of Performance Indicators Relationships on Sustainable Healthcare Supply Chain Performance Measurement Using Balanced Scorecard and DEMATEL
}

\author{
Eko Budi Leksono ${ }^{\# *}$, Suparno*, Iwan Vanany* \\ \# Department of Industrial Engineering, University of Muhammadiyah Gresik, Jl. Sumatera 101 GKB, Gresik, 61121, Indonesia \\ E-mail:eko_budileksono@umg.ac.id
}

*Department of Industrial Engineering, Institut Teknologi Sepuluh Nopember, Kampus ITS Sukolilo, Surabaya, 60111, Indonesia

\begin{abstract}
Sustainable healthcare supply chain performance measurement (SHSCPM) concept is still less developed. Globalization and pressure from stakeholder demand the operation of the supply chain to give attention to the environment effect, community, economic and intangibility assets. SHSCPM is feasibly developed for measuring the performance of simultaneous sustainability aspects and intangibility assets to meet customer satisfaction. This article discusses SHSCPM based on the balanced scorecard (BSC) with attention to the sustainability aspects, intangibility assets and relationships between the performance of perspectives and indicators. The perspectives and indicators of performance were identified by literature and the confirmed and validated by the survey to 7 expert respondents. We found 5 perspectives and 39 indicators from literature which were then confirmed to expert through a survey with an in-depth interview. From a survey that validated with a weighted average (WA) and level of consensus (LC), we found 31 valid indicators. Finally, 29 indicators from DEMATEL process were selected to be used on SHSCPM. The DEMATEL process found 2 indicators aren't important and influence for other, namely inventory cost and regulations and laws. Besides, the four results on this study: intangibility indicators incorporated on innovation and growth were most affect to other indicators which the intangibility indicators were related with human resource, indicators on customer perspective were most important compared to other indicators, indicators on economic aspect were most important compared indicators on environmental and social aspects, and indicators on social aspect were not affected by other indicators. After that, human resource and customer were main factors for SHSCPM. Finally, relationships between perspectives and indicators used to design of BSC strategy map.
\end{abstract}

Keywords - SHSCPM; weighted average; level of consensus; balanced scorecard; DEMATEL

\section{INTRODUCTION}

The service supply chain (SSC) implementation has become a trend since service sector gives a significant contribution to the gross domestic product (GDP) [1]-[3] and affects' global economy [4]. In 2013, services sector contribution to Indonesia GDP in 2013 was $39.87 \%$ [5]. During 2012 - 2015, the services sector contribution to Product Domestic Regional Brute (PDRB) of East Java Province has an average of $71.11 \%$. The sector's contribution has increasing trends are tourism, hotel \& restaurant, professional services, banking \& insurance and healthcare [6]. The healthcare sector is feasible to develop in Indonesia, especially in East Java Province. Implementation of the supply chain and performance measurement can be used to develop the healthcare performance. [7]. The healthcare supply chain (HSC) implementation is part of
SSC which healthcare need an affiliation with all SC actors to meet customer satisfaction [1].

Globalization and pressure from stakeholder demand the operation of SSC to pay attention to sustainability issues, which consists of economic, social and environmental issues simultaneously [8]. The implementation of sustainable SSC can minimize the negative effect of the operation on the environment and social and maximize profit [9]. The sustainable HSC (SHSC) concept hasn't been developed so there are can be research opportunities in the future.

The concept of SHSC performance measurement (SHSCPM) has not been developed, and there are still oriented to aspects of economic and environmental (green), such as environmental SCPM [10], life cycle assessment and life cost assessment [11]. Sustainable SC performance defines as a company's capacity to reduce the use of materials, energy, or water and to find more eco-efficient solutions by improving supply chain [12]. Publications of SHSCPM concept still less and there is less of simultaneous 
integration between aspects of economic, environmental and social. Furthermore, SHSCPM research is feasible to develop in Indonesia. Besides, the main characteristic of services sector are intangibility assets which related with human resources [13]-[16], so the SHSCPM implementation must attend intangibility assets on coordination process, performance measurement and customer relationship to meet the customer satisfaction.

This article discusses the relationship between perspectives and indicators on the SHSCPM. The balanced scorecard (BSC) performance measurement method will combine with the decision-making trial and evaluation laboratory (DEMATEL) to relationships design between perspectives and indicators of performance. Finally, combine BSC and DEMATEL were used to determine the level of importance and influence of the perspectives and indicators on SHSCPM.

\section{MATERIAL AND METHOD}

\section{A. Literature Review}

1) Healthcare SCPM (HSCPM) and Sustainable $H S C P M(S H S C P M)$

The actors on HSC are a producer, supplier (purchaser), healthcare provider and patient [17], [18]. The HSCPM concepts and performance orientation, include continuous improvement and customer satisfaction [19], information technology, demand, customer relationship, supplier relationship, capacity \& resources [7], trust, knowledge exchange, IT integration between supplier and service provider [20], costs and benefit [21].

The SHSCPM concept still less and there is few of simultaneous integration between aspects of economic, environmental and social. Some concept of sustainable SSC more explores of economic and environmental aspect [22], [23], including on health care. The SHSCPM need new concept that can integrate dimensions of economic, environmental and social simultaneously

2) Balanced Scorecard (BSC) and the Decision-Making Trial and Evaluation Laboratory (DEMATEL)

$\mathrm{BSC}$ is a performance measurement model that describes the relationship between perspectives and or indicators on BSC strategy map as a business strategy. The strategy map is a business strategy that is related to financial and nonfinancial execution. BSC is a dynamic and innovative performance measurement method that can be developed and collaborated with other methods [24]. Given the BSC flexibility, the SHSCPM can adopt BSC [25], [26]. Determination of performance perspectives is the starting point of BSC because the perspectives explain viewpoint of organization performance. There are five perspectives used in services sector performance: finance, customer, operation, information and innovation \& growth [27].

DEMATEL is a multiple-attribute decision making (MADM) method that is used as a tool to help in decision making [28]. DEMATEL is used to analyse the component structure from decision variables. DEMATEL can be used to view the direct or indirect relationship between variable or attribute [29]. Besides, DEMATEL can describe the level of important and level of influence of an attribute or a variable on the system, DEMATEL uses matrix system to get all causal relationships of an attribute or a variable with the others [29]. DEMATEL can integrate with BSC for build a strategy map while DEMATEL can describe the relationship between performance indicators.

\section{3) Performance Indicator of SHSCPM}

Performance indicators of SHSCPM were taken from the literature based on healthcare topics: performance measurement (PM), SSC, SSCPM and sustainable SSC.

The indicators listed in Table 1 may be used to develop SHSCPM model. Before used, the indicators must be validated by the survey to all HSC actors.

\section{B. Method}

Development of the model that was used survey with an in-depth interview. In this study, we asked one pharmaceutical industry manager, one supplier owner, two private hospital professional managers, one public hospital professional manager, and two clinical professional managers. Stages of developing the model are preliminary study, determine the relationship between perspectives and indicators, and strategy map.

\section{1) Preliminary Study}

The indicators from literature review confirmed to respondents through a survey. Survey validation use weighted average (WA) and level of consensus (LC). Steps on preliminary study [54]:

- Survey to identify important level of indicators with Likert Scale ( 1 = very unimportant, $2=$ unimportant, $3=$ neutral, $4=$ important, $5=$ very important) .

- Validation of survey results by calculating WA and LC using the cut off : economic aspect (WA $\geq 4.2 \&$ $\mathrm{LC} \geq 0.5$ ), environmental and social aspects (WA $\geq$ 4.5 and $\mathrm{LC} \geq 0.7$ ).

$$
\begin{aligned}
& W A_{n}=\frac{\sum\left(R_{i} x L S\right)}{\sum R} \\
& L C_{n}=\frac{\sum(L S)}{\sum R}
\end{aligned}
$$

Where, $\mathrm{R}_{\mathrm{i}}=$ respondent $\mathrm{i}$,

$\mathrm{LS}=$ Likert score with value 4 (important) and 5 (very important),

$\mathrm{R}=$ total of respondents

- Classifying valid indicators based on performance perspectives: financial, customer, operational, information and innovation and growth

- The Relationship between Perspectives and Indicators

This stage using DEMATEL method [29] with steps:

- Survey to identify level of influence between perspective and indicator with scale: $0-4(0=$ no influence, 1 = low, $2=$ normal, $3=$ strong, $4=$ very strong).

- Building direct relation matrix (A) based on the average of influence value from $a_{i}$ to $a_{j}$. 
TABLE I

PERFORMANCE INDICATORS FOR SHSCPM

\begin{tabular}{|c|c|c|}
\hline No & Indicators & References \\
\hline & Economic & \\
\hline 1 & Patient (demand) & {$[1],[7],[30]-[32]$} \\
\hline 2 & Human resource (HR) & {$[1],[21],[33]$} \\
\hline 3 & $\begin{array}{l}\text { Capacity \& } \\
\text { professionalism }\end{array}$ & $\begin{array}{l}{[1],[7],[21],[30],[34]-} \\
{[37]}\end{array}$ \\
\hline 4 & Effectivity & $\begin{array}{l}{[1],[10],[26],[30],[36],} \\
{[38]-[42]}\end{array}$ \\
\hline 5 & Inventory level & $\begin{array}{l}{[17],[31],[32], \quad[39],} \\
{[43],[44]}\end{array}$ \\
\hline 6 & Tariff of service & {$[17],[21],[34],[38],[44]$} \\
\hline 7 & Quality of service & $\begin{array}{l}{[17],[21],[30],[34],} \\
{[36]-[38],[40],[43]-[46]}\end{array}$ \\
\hline 8 & Standard of service & {$[21],[35],[45],[47],[48]$} \\
\hline 9 & Return of Investment & {$[49],[50]$} \\
\hline 10 & Return of Assets & [49], [50] \\
\hline 11 & Efficiency & $\begin{array}{l}{[21],[26], \quad[30], \quad[36],} \\
{[42],[43]}\end{array}$ \\
\hline 12 & Profit & [11] \\
\hline 13 & Inventory cost & [17], [21], [48] \\
\hline 14 & Revenue & {$[26],[49]$} \\
\hline 15 & Innovation & {$[26],[30]$} \\
\hline 16 & Flexibility & {$[36],[51]$} \\
\hline 17 & Suppliers timeliness & {$[1],[38]$} \\
\hline 18 & Commitment to supplier & $\begin{array}{l}{[7],[10],[21],[34],[43],} \\
{[47],[52],[53]}\end{array}$ \\
\hline 19 & $\begin{array}{l}\text { Integration of information } \\
\text { system }\end{array}$ & [20], [41], [50], [52] \\
\hline 20 & Delivery & {$[32],[40],[45],[47]$} \\
\hline 21 & Education and training & {$[10],[26],[34],[35],[38]$} \\
\hline 22 & Research \& Development & {$[10]$} \\
\hline & Environmental & \\
\hline 23 & Green technology & {$[10],[45]$} \\
\hline 24 & Green material & [10], [45] \\
\hline 25 & $\begin{array}{l}\text { Partnership with green } \\
\text { organization }\end{array}$ & {$[10]$} \\
\hline 26 & Waste treatment & {$[10],[51]$} \\
\hline 27 & $\begin{array}{l}\text { Environmental } \\
\text { certifications }\end{array}$ & {$[10]$} \\
\hline 28 & Work physic environment & {$[37]$} \\
\hline & Social & \\
\hline 29 & Accessibility & [37] \\
\hline 30 & Regulations and laws & $\begin{array}{l}{[1],[17],[26],[30],[34],} \\
{[37],[38],[42],[45]-[47]}\end{array}$ \\
\hline 31 & Customer satisfaction & $\begin{array}{l}{[1],[7],[33],[35],[44],} \\
{[47],[52]}\end{array}$ \\
\hline 32 & Customer service & {$[1]$} \\
\hline 33 & $\begin{array}{l}\text { Medical information } \\
\text { system }\end{array}$ & {$[1],[20],[45],[47],[53]$} \\
\hline 34 & Patient loyalty & $\begin{array}{l}{[1],[20],[39],[50],[52],} \\
{[53]}\end{array}$ \\
\hline 35 & $\begin{array}{l}\text { Collaboration with } \\
\text { supplier }\end{array}$ & {$[17],[26],[34],[42],[46]$} \\
\hline 36 & Stakeholder satisfaction & $\begin{array}{l}{[11],[21],[26],[30],} \\
{[35],[36],[42],[47]}\end{array}$ \\
\hline 37 & Health \& safety & $\begin{array}{l}{[11],[21],[26],[30],} \\
{[35],[36],[42],[47]}\end{array}$ \\
\hline 38 & $\begin{array}{l}\text { Sharing of information \& } \\
\text { knowledge }\end{array}$ & {$[20],[44],[50],[53]$} \\
\hline 39 & Organization behaviour & [34], [36], [37] \\
\hline
\end{tabular}

$$
A=\left[a_{i j}\right]_{n x n}
$$

- $\quad$ Building normalization matrix $(\mathrm{X})=\mathrm{A} \times \mathrm{s}$

$$
s=\min \left[\frac{1}{\max \sum_{j=1}^{n} a_{i j}}, \frac{1}{\max \sum_{i=1}^{n} a_{i j}}\right]
$$

- Building total relationship matrix (T), with I as the identity matrix.

$$
T=X(I-X)^{-1}
$$

- Calculation of the level of importance $(D+R)$ and influence ( $-\mathrm{R})$ between perspectives and indicators.

$$
\text { Element of } \mathrm{T}=\left[t_{i j}\right]_{n x n}, i, j=1,2, \ldots, n
$$

Where, $i=$ rows, $j=$ column.

Then, calculate $\mathrm{D}$ and $\mathrm{R}$ that represent of direct and indirect relationship from row and column [29].

$$
\begin{gathered}
D=\left[\sum_{j=1}^{n} t_{i j}\right]_{n x 1} \\
R=\left[\sum_{i=1}^{n} t_{i j}\right]_{n x 1},(i, j=1,2, \ldots, n)
\end{gathered}
$$

- Building significant matrix

The significant matrix used to describe the relationship between perspectives and indicators. Steps in the significant matrix:

- Calculate the average of $\mathrm{T}$ :

$$
\bar{X}=\frac{\sum T}{\sum i j}
$$

Where, $i=$ sum of row and $j=$ sum of column

- Reduction of all $\mathrm{T}$ with $\bar{X}$ or $(\mathrm{T}-\bar{X})$, a value of significant matrix indicates the level of relationship.

- After significant matrix has made, so performance indicators can be classified based on perspectives of BSC

2) Design of BSC Strategy Map

BSC strategy maps designed based on important level, influence level, and significance matrix. The indicator with a low level of important and influence can be ignored on designing of BSC strategy map.

\section{RESULTS AND DISCUSSION}

\section{A. Validation and Classification of Indicators}

Based on validation with WA and LC, we found eight not valid indicators. Not valid indicators indicate that indicator not important. Not valid indicators shown in Table 2. 
TABLE II

INDICATOR VALIDATION

\begin{tabular}{|l|c|c|c|}
\hline $\begin{array}{c}\text { Sustainability dimensions \& } \\
\text { performance indicators }\end{array}$ & WA & LC & Note \\
\hline Economic aspect & & & \\
\hline Human resource (HR) & 2.57 & 0.28 & Not Valid \\
\hline Tariffs & 2.57 & 0.28 & Not Valid \\
\hline Return of Investment & 2.66 & 0.28 & Not Valid \\
\hline Return of Assets & 2.57 & 0.28 & Not Valid \\
\hline Commitment to supplier & 2.71 & 0.28 & Not Valid \\
\hline Environmental aspects & & & \\
\hline $\begin{array}{l}\text { Partnership with green } \\
\text { organization }\end{array}$ & 2.71 & 0.42 & Not Valid \\
\hline Social aspects & & & \\
\hline Accessibility & 3.42 & 0.57 & Not Valid \\
\hline Customer service & 3.42 & 0.47 & Not Valid \\
\hline
\end{tabular}

Then, valid indicator classified based on the performance perspectives. Classification indicator to the performance perspectives was done by survey with an in-depth interview with expert respondents. Indicators classification is shown in Table 3.

TABLE III

INDICATOR CLASSIFICATION BASED ON PERSPECTIVE AND SUSTAINABILITY

\begin{tabular}{|c|c|c|c|}
\hline \multirow[t]{2}{*}{ Perspective } & \multicolumn{3}{|c|}{ Sustainability dimensions } \\
\hline & Economic & Environmental & Social \\
\hline Financial & $\begin{array}{l}\text { Patient } \\
\text { demand } \\
\text { (X1) } \\
\text { Effectivity } \\
\text { (X2) } \\
\text { Efficiency } \\
\text { (X3) } \\
\text { Profit (X4) } \\
\text { Inventory } \\
\text { cost (X5) } \\
\text { Revenue } \\
\text { (X6) }\end{array}$ & & \\
\hline Customer & $\begin{array}{l}\text { Quality of } \\
\text { service } \\
\text { (X7) } \\
\text { Delivery } \\
\text { (X8) }\end{array}$ & & $\begin{array}{l}\text { Customer } \\
\text { satisfaction } \\
\text { (X9) } \\
\text { Patient loyalty } \\
\text { (X10) } \\
\text { Stakeholder } \\
\text { satisfaction } \\
\text { (X11) }\end{array}$ \\
\hline Operational & $\begin{array}{l}\text { Inventory } \\
\text { level (X12) } \\
\text { Standard of } \\
\text { service } \\
\text { (X13) } \\
\text { Flexibility } \\
\text { (X14) } \\
\text { Suppliers } \\
\text { timeliness } \\
\text { (X15) }\end{array}$ & $\begin{array}{l}\text { Green } \\
\text { technology } \\
\text { (X16) } \\
\text { Green material } \\
\text { (X17) } \\
\text { Waste treatment } \\
\text { (X18) } \\
\text { Work physic } \\
\text { environment } \\
\text { (X19) }\end{array}$ & $\begin{array}{l}\text { Collaboration } \\
\text { with supplier } \\
(\mathrm{X} 20)\end{array}$ \\
\hline Information & $\begin{array}{l}\text { Integration } \\
\text { of inform. } \\
\text { system }(X 21)\end{array}$ & $\begin{array}{l}\text { Environmental } \\
\text { certifications } \\
\text { (X22) }\end{array}$ & $\begin{array}{l}\text { Medical } \\
\text { inform. system } \\
\text { (X23) } \\
\text { Sharing inform. } \\
\text { \& knowledge } \\
\text { (X24) }\end{array}$ \\
\hline
\end{tabular}

\begin{tabular}{|c|c|c|}
\hline $\begin{array}{l}\text { Innovation } \\
\& \text { growth }\end{array}$ & $\begin{array}{l}\text { Capacity \& } \\
\text { professiona } \\
\text { lism (X25) } \\
\text { Innovation } \\
\text { (X26) } \\
\text { Education } \\
\& \quad \text { training } \\
(\mathrm{X} 27) \\
\mathrm{R} \quad \& \quad \mathrm{D} \\
(\mathrm{X} 28)\end{array}$ & $\begin{array}{l}\text { Regulations \& } \\
\text { laws (X29) } \\
\text { Health \& } \\
\text { safety (X30) } \\
\text { Organization } \\
\text { behaviour } \\
\text { (X31) }\end{array}$ \\
\hline
\end{tabular}

\section{B. Level of Importance and Influence}

The DEMATEL stages were used to make relationship started from building the direct relationship matrix based on average values from respondents and finished at establishing a relationship between perspectives and indicators.

Based on direct relationship matrix, we make the total relationship matrix that indicates the level of importance and influence of perspectives and or indicators.

Table 4 show total relationship matrix of perspectives. Besides, Table 4 shows the customer perspective was most important compared to other perspectives $(\mathrm{D}+\mathrm{R}=7.898)$, and the innovation and growth were a most influential to other perspectives $(\mathrm{D}-\mathrm{R}=1.371)$. The innovation and growth related to human resource, so the existence of human resource was the most influence on SHSCPM.

TABLE IV

TOTAL RElATIONSHIP MATRIX (T) OF PERSPECTIVES

\begin{tabular}{|l|c|c|c|c|}
\hline \multicolumn{1}{|c|}{ Perspectives } & D & R & D + R & D - R \\
\hline Financial & 3.27 & 3.756 & 7.026 & -0.485 \\
\hline Customer & 3.715 & 4.183 & 7.898 & -0.468 \\
\hline Operational & 3.518 & 3.924 & 7.442 & -0.405 \\
\hline Information & 3.181 & 3.194 & 6.375 & -0.013 \\
\hline Innovation \& Growth & 4.421 & 3.05 & 7.471 & 1.371 \\
\hline
\end{tabular}

Table 5 show total relationship matrix of indicators based on sustainability. Based on sustainability, All of the indicators have $(\mathrm{D}+\mathrm{R})$ positive value which indicates all indicator was important on SHSCPM. Besides, indicators incorporated on economic aspect were most important (D + $\mathrm{R}=5.44$ ) compared other indicators incorporated on the social and environmental aspects. Indicators incorporated on environmental aspects were the most influence $(\mathrm{D}-\mathrm{R}=$ $0.15)$ to other indicators. Indicators incorporated on the social lowest influence to other indicators $(D-R=-0.12)$.

Then, total relationship matrix based on sustainability can change based on performance perspectives. Table 6 show total relationship matrix of indicators based on perspectives.

Table 6 show indicators incorporated on innovation and growth were most important $(\mathrm{D}+\mathrm{R}=5.64)$ and most influence to other indicators $(\mathrm{D}-\mathrm{R}=0.44$ ). So, human resource is very important and very influential on SHSCPM. 
TABLE V

TOTAL RELATIONSHIP MATRIX (T) OF INDICATOR BASED ON

SUSTAINABILITY

\begin{tabular}{|c|c|c|c|c|c|}
\hline Aspects & Indicators & $\mathbf{D}+\mathbf{R}$ & $\mathbf{D}-\mathbf{R}$ & $\dot{\mathbf{X}}_{\mathrm{D}+\mathrm{R}}$ & $\dot{\mathbf{X}}_{\text {D-R }}$ \\
\hline \multirow{17}{*}{ Economic } & Patient (demand) & 5.94 & -0.28 & \multirow{17}{*}{5.44} & \multirow{17}{*}{0.02} \\
\hline & $\begin{array}{l}\text { Capacity \& } \\
\text { professionalism }\end{array}$ & 6.26 & 0.28 & & \\
\hline & Effectivity & 5.6 & -0.2 & & \\
\hline & Inventory level & 4.99 & 0.1 & & \\
\hline & Quality of service & 6.41 & 0.1 & & \\
\hline & $\begin{array}{l}\text { Standard of } \\
\text { service }\end{array}$ & 5.82 & 0.18 & & \\
\hline & Efficiency & 5.1 & -0.12 & & \\
\hline & Profit & 5.54 & -0.84 & & \\
\hline & Inventory cost & 3.13 & -0.65 & & \\
\hline & Revenue & 5.03 & -0.76 & & \\
\hline & Innovation & 6.68 & 0.53 & & \\
\hline & Flexibility & 5.86 & 0.22 & & \\
\hline & $\begin{array}{l}\text { Suppliers } \\
\text { timeliness }\end{array}$ & 4.46 & 0.52 & & \\
\hline & $\begin{array}{l}\text { Integration of } \\
\text { information } \\
\text { system }\end{array}$ & 5.41 & 0.16 & & \\
\hline & Delivery & 4.81 & -0.17 & & \\
\hline & $\begin{array}{l}\text { Education \& } \\
\text { training }\end{array}$ & 5.72 & 0.77 & & \\
\hline & R \& D & 5.74 & 0.52 & & \\
\hline \multirow{5}{*}{$\begin{array}{l}\text { Environ } \\
\text { mental }\end{array}$} & Green technology & 5.38 & 0.51 & \multirow{5}{*}{4.87} & \multirow{5}{*}{0.15} \\
\hline & Green materials & 4.38 & 0.2 & & \\
\hline & Waste treatment & 5.16 & -0.07 & & \\
\hline & $\begin{array}{l}\text { Work physic } \\
\text { environment }\end{array}$ & 4.75 & 0.22 & & \\
\hline & $\begin{array}{l}\text { Environmental } \\
\text { certifications }\end{array}$ & 4.71 & -0.08 & & \\
\hline \multirow{9}{*}{ Social } & $\begin{array}{l}\text { Regulations \& } \\
\text { laws }\end{array}$ & 4.16 & 0.01 & \multirow{9}{*}{5.16} & \multirow{9}{*}{-0.12} \\
\hline & $\begin{array}{l}\text { Customer } \\
\text { satisfaction }\end{array}$ & 5.27 & -0.87 & & \\
\hline & $\begin{array}{l}\text { Medical inform. } \\
\text { system }\end{array}$ & 5.29 & -0.02 & & \\
\hline & Patient loyalty & 4.62 & -0.95 & & \\
\hline & $\begin{array}{l}\text { Collaboration } \\
\text { with supplier }\end{array}$ & 5.87 & 0.15 & & \\
\hline & $\begin{array}{l}\begin{array}{l}\text { Stakeholder } \\
\text { satisfaction }\end{array} \\
\end{array}$ & 5.57 & -0.78 & & \\
\hline & Health \& safety & 4.57 & 0.35 & & \\
\hline & $\begin{array}{l}\text { Sharing inf. \& } \\
\text { knowledge }\end{array}$ & 4.74 & 0.34 & & \\
\hline & $\begin{array}{l}\text { Organization } \\
\text { behaviour }\end{array}$ & 6.34 & 0.65 & & \\
\hline
\end{tabular}

TABLE VI

TOTAL RELATIONSHIP MATRIX OF INDICATOR BASED ON PERSPECTIVE

\begin{tabular}{|c|c|c|c|c|c|}
\hline Perspective & Indicators & $\mathbf{D}+\mathbf{R}$ & D-R & $\dot{\mathbf{X}}_{\mathrm{D}+\mathbf{R}}$ & $\dot{\mathbf{X}}_{\text {D-R }}$ \\
\hline \multirow{6}{*}{ Financial } & Patient (demand) & 5.94 & -0.27 & \multirow{6}{*}{5.06} & \multirow{6}{*}{-0.47} \\
\hline & Effectively & 5.6 & -0.21 & & \\
\hline & Efficiency & 5.1 & -0.12 & & \\
\hline & Profit & 5.54 & -0.84 & & \\
\hline & Inventory cost & 3.13 & -0.64 & & \\
\hline & Revenue & 5.03 & -0.76 & & \\
\hline \multirow[t]{3}{*}{ Customer } & $\begin{array}{l}\text { Quality of } \\
\text { service }\end{array}$ & 6.41 & 0.1 & \multirow{3}{*}{5.33} & \multirow{3}{*}{-0.53} \\
\hline & Delivery & 4.8 & -0.17 & & \\
\hline & $\begin{array}{l}\text { Customer } \\
\text { satisfaction }\end{array}$ & 5.27 & -0.87 & & \\
\hline
\end{tabular}

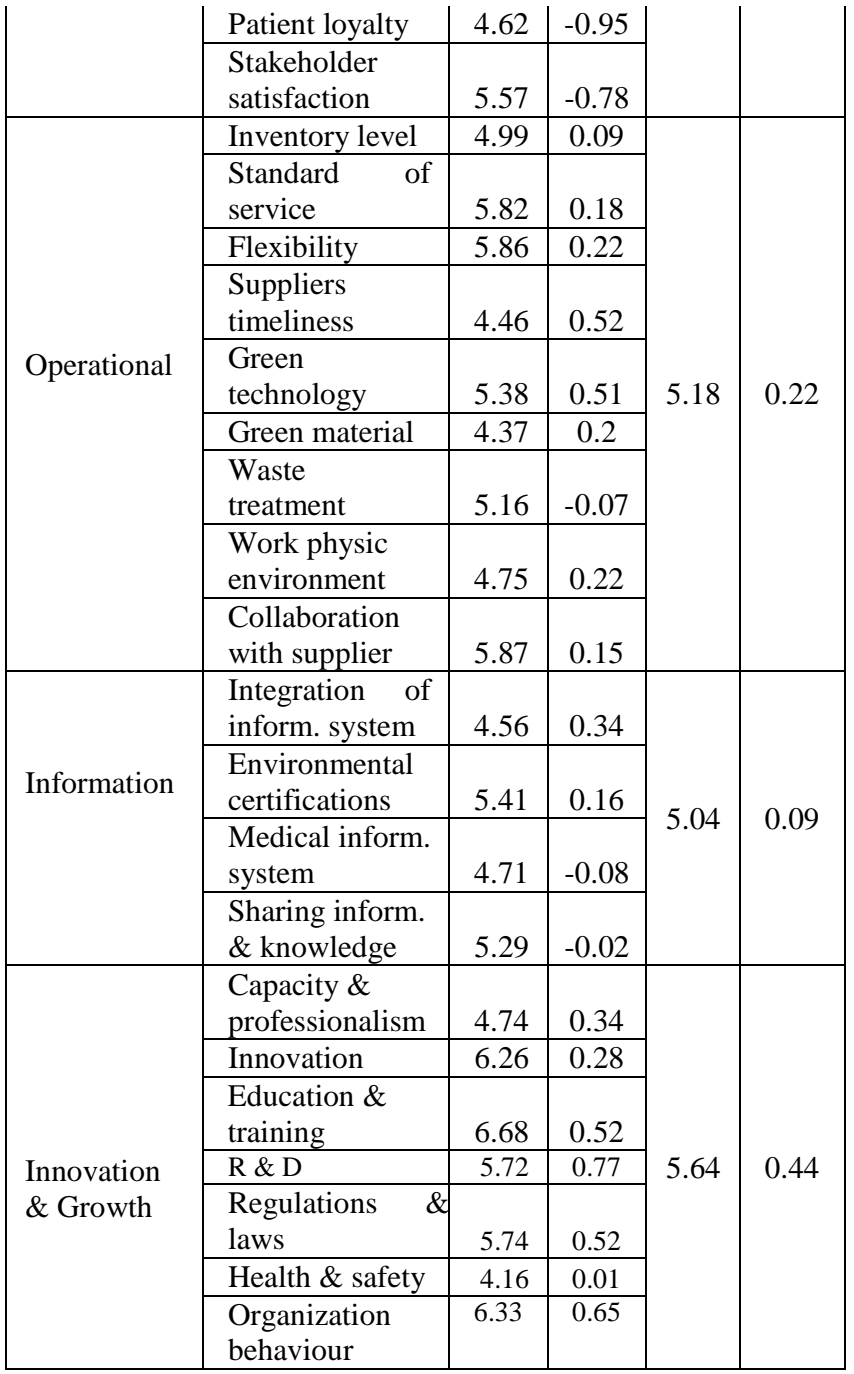

\section{Relationship between Perspectives and Indicators}

The relationship between perspectives and indicators can be designed based on the significant matrix. The significant matrix can be built based on total relationship matrix. Stage on build significant matrix for perspectives:

- Calculate the average of $\mathrm{T}$ :

$$
\bar{X}=\frac{18.1}{25}=0.724
$$

- Reduction of all T with 0.724. A value of significant matrix indicates the level of relationship. A minus (-) value indicates that there is no relationship between perspectives. Equally, a positive (+) value indicates that a row perspective has relation with a column perspective.

Table 7 shows significance matrix of perspectives. The same stage can be used to build of the significant matrix for indicators. 
TABLE VII

SIGNIFICANT MATRIX FOR PERSPECTIVES

\begin{tabular}{|c|c|c|c|c|c|}
\hline & F & C & O & I & IG \\
\hline F & -0.165 & 0.0157 & -0.023 & -0.174 & -0.147 \\
\hline C & 0.108 & -0.058 & 0.056 & -0.067 & -0.088 \\
\hline O & 0.036 & 0.105 & -0.138 & -0.138 & -0.112 \\
\hline I & -0.071 & 0.043 & 0.0038 & -0.308 & -0.253 \\
\hline I G & 0.226 & 0.276 & 0.2248 & 0.078 & -0.15 \\
\hline
\end{tabular}

$\mathrm{F}=$ Financial, $\mathrm{C}=$ Customer, $\mathrm{O}=$ Operational,

$\mathrm{I}=$ Information, $\mathrm{IG}=$ Innovation and Growth
After building of the significant matrix, so BSC strategy map maybe design. From to significant matrix for indicators, the existence of inventory cost $\left(\mathrm{X}_{5}\right)$ and regulations \& laws $\left(\mathrm{X}_{29}\right)$ are not important and do not have significant relationships with other, so inventory cost and regulations \& laws can be ignored on BSC strategy map. Fig. 1 shows BSC strategy map with twenty-nine indicators on five perspectives.

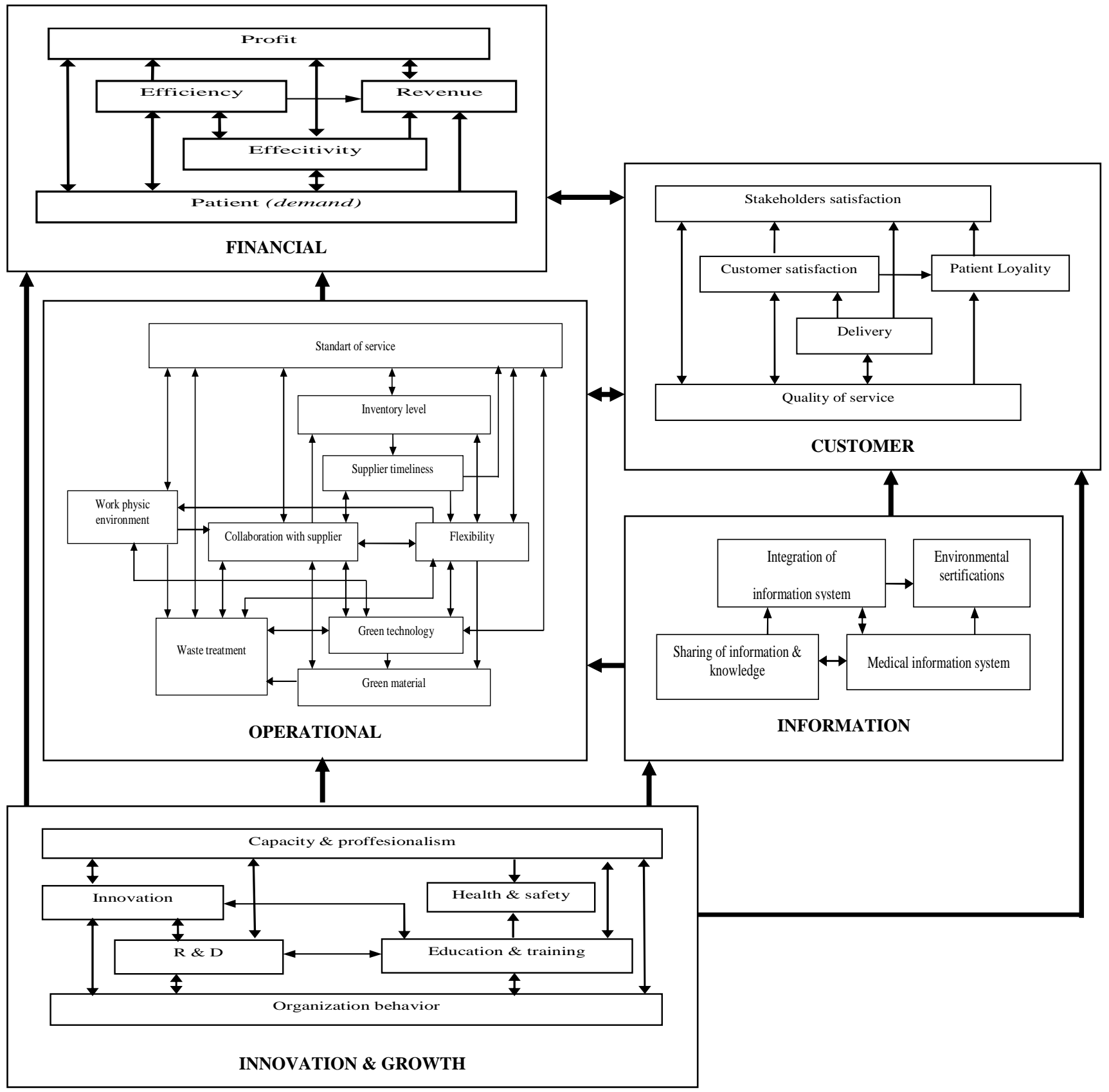

Fig. 1 BSC strategy map 


\section{CONCLUSIONS}

The design of SHSCPM based on BSC and DEMATEL used five performance perspectives with twenty-nine indicators. The indicators attend to sustainability aspects and intangibility assets that relate to human resource. The twenty-nine indicators are appointed from literature review, validation of survey result with WA and LC, and DEMATEL significant matrix.

From DEMATEL process, we found four results on SHSCPM: First, intangibility indicators incorporated on innovation and growth were most affect to other indicators which the intangibility indicators were related to human resource. Second, indicators on customer perspective were most important compared to other indicators, indicators incorporated on economic aspect were most important compared indicators on environmental and social aspects. Furthermore, human resource and customer were main factors for SHSCPM. Third, indicators on social aspect were not affected to other indicators. Finally, relationships between perspectives and indicators used to design of BSC strategy map. Design of SHSCPM can be ignored of indicators of inventory cost and regulations and laws because the indicators not important and do not have the effect on other indicators.

The limitation of the research, the model is a framework of the SHSCPM. There is need implementation in a healthcare service provider.

Further research needs calculate of indicators weight with attending the relationship between indicators by analytical network process (ANP). Finally, the simulation by system dynamic may be used to predict of sustainable HSC performance in the future.

\section{REFERENCES}

[1] T. Baltacioglu, E. Ada, M. D. Kaplan, O. Yurt And, and Y. Cem Kaplan, "A New Framework for Service Supply Chains," Serv. Ind. J., vol. 27, no. 2, pp. 105-124, 2007.

[2] M. Giannakis, "Conceptualizing and managing service supply chains," Serv. Ind. J., vol. 31, no. 11, pp. 1809-1823, 2011.

[3] J. Drzymalski, "Supply Chain Frameworks for the Service Industry: A Review of the Literature," vol. 1, pp. 31-42, Dec. 2012.

[4] D. W. Cho, Y. H. Lee, S. H. Ahn, and M. K. Hwang, "A framework for measuring the performance of service supply chain management," Comput. Ind. Eng., vol. 62, no. 3, pp. 801-818, 2012.

[5] BPS, "Pertumbuhan Ekonomi Indonesia 2013," Berita Resmi Statistik, no. 16/02/Th. XVII, 201, pp. 1-9,2014.

[6] BPS, Jawa Timur Dalam Angka, 2015.

[7] L. L. Yap and C. L. Tan, "The Effect of Service Supply Chain Management Practices on the Public Healthcare Organizational Performance," vol. 3, no. 16, pp. 216-224, 2012.

[8] E. Hassini, C. Surti, and C. Searcy, "A literature review and a case study of sustainable supply chains with a focus on metrics," Int. J. Prod. Econ., vol. 140, no. 1, pp. 69-82, 2012.

[9] X. Xu and D. Gursoy, "A Conceptual Framework of Sustainable Hospitality Supply Chain Management," J. Hosp. Mark. Manag., pp. 1-31, 2014.

[10] P. Chithambaranathan, N. Subramanian, and A. Gunasekaran, "Int . J . Production Economics Service supply chain environmental performance evaluation using grey based hybrid MCDM approach," Intern. J. Prod. Econ., no. 2006, pp. 1-14, 2015.

[11] S. Unger and A. Landis, "Assessing the environmental, human health, and economic impacts of reprocessed medical devices in a Phoenix hospital's supply chain," J. Clean. Prod., vol. 112, pp. 1995-2003, 2016.
[12] E. Ortas, J. M. Moneva, and I. Álvarez, "Sustainable supply chain and company performance: A global examination," Supply Chain Manag. An Int. J., vol. 19, no. 3, pp. 332-350, 2014.

[13] B. Marr and C. Adams, "The balanced scorecard and intangible assets : similar ideas , unaligned concepts," Meas. Bus. Excell., vol. 8, no. 3, pp. 18-27, 2006.

[14] K. Zigan, F. Macfarlane, and T. Desombre, "Intangible resources as performance drivers in European hospitals," vol. 57, no. 1, pp. 57-71, 2008.

[15] K. Zigan and D. Zeglat, "Intangible resources in performance measurement systems of the hotel industry," Facilities, vol. 28, no. 13/14, pp. 597-610, 2010.

[16] S. A. Melnyk, U. Bititci, K. Platts, J. Tobias, and B. Andersen, "Is performance measurement and management fit for the future?," Manag. Account. Res., vol. 25, no. 2, pp. 173-186, 2014.

[17] B. K. Smith, H. Nachtmann, and E. A. Pohl, "Quality Measurement in the Healthcare Supply Chain," Qual. Manag. J., vol. 18, no. 4, pp. $50-60,2011$.

[18] S. Priyan and R. Uthayakumar, "Optimal inventory management strategies for pharmaceutical company and hospital supply chain in a fuzzy-stochastic environment," Oper. Res. Heal. Care, vol. 3, no. 4, pp. 177-190, Dec. 2014.

[19] K. Swinehart and A. E. Smith, "Internal supply chain performance measurement: a health care continuous improvement implementation.," Int. J. Health Care Qual. Assur. Inc. Leadersh. Health Serv., vol. 18, no. 6-7, pp. 533-42, Jan. 2005.

[20] D. Q. Chen, D. S. Preston, and W. Xia, "Enhancing hospital supply chain performance: A relational view and empirical test," J. Oper. Manag., vol. 31, no. 6, pp. 391-408, Sep. 2013.

[21] F. Lega, M. Marsilio, and S. Villa, "An evaluation framework for measuring supply chain performance in the public healthcare sector: evidence from the Italian NHS," Prod. Plan. Control, vol. 24, no. 10-11, pp. 931-947, 2013.

[22] M. Varsei, C. Soosay, B. Fahimnia, and J. Sarkis, "Framing sustainability performance of supply chains with multidimensional indicators," Supply Chain Manag. An Int. J., vol. 19, no. 3, pp. 242257, 2014.

[23] P. Beske-janssen, M. P. Johnson, and S. Schaltegger, "20 years of performance measurement in sustainable supply chain management what has been achieved ?," Supply Chain Manag. An Int. J., vol. 20, no. 6, pp. 664-680, 2015.

[24] R. S. Kaplan and D. P. Norton, "Response to S. Voelpel et al., 'The tyranny of the Balanced Scorecard in the innovation economy,' Journal of Intellectual Capital, Vol. 7 No. 1, 2006, pp. 43-60," $J$. Intellect. Cap., vol. 7, no. 3, pp. 421-428, 2006.

[25] K. E. Voelker, J. S. Rakich, and G. R. French, "The balanced scorecard in healthcare organizations: A performance measurement and strategic planning methodology," Hosp. Top., vol. 79, no. 3, pp. 13-24, 2001.

[26] A. M. Wicks, L. S. Clair, and K. Charles S, "Competing Values in Healthcare: Balancing the ( Un ) Balanced Scorecard," J. Healthc. Manag., vol. 52, no. 5, pp. 309-324, 2007.

[27] E. B. Leksono, Suparno, and I. Vanany, "Development of Conceptual Framework for Sustainable Supply Chain Performance Measurement in Service Industries," in 8th Widyatama International Seminar on Sustainability 2016, paper 27, pp. 142.

[28] I. Gölcük and A. Baykasoglu, "An analysis of DEMATEL approaches for criteria interaction handling within ANP," Expert Syst. Appl., vol. 46, pp. 346-366, 2016.

[29] F. Chen, T. Hsu, and G. Tzeng, "A balanced scorecard approach to establish a performance evaluation and relationship model for hot spring hotels based on a hybrid MCDM model combining DEMATEL and ANP," Int. J. Hosp. Manag., vol. 30, no. 4, pp. 908932, 2011.

[30] S. Purbey, K. Mukherjee, and C. Bhar, "Performance measurement system for healthcare processes," Int. J. Product. Perform. Manag., vol. 56, no. 3, pp. 241-251, 2007.

[31] C. Samuel, K. Gonapa, P. K. Chaudhary, and A. Mishra, "Supply chain dynamics in healthcare services.," Int. J. Health Care Qual. Assur., vol. 23, no. 7, pp. 631-42, 2010.

[32] S. M. Zahraee, J. M. Rohani, A. Firouzi, and A. Shahpanah, "Efficiency improvement of blood supply chain system using Taguchi method and dynamic simulation," Procedia Manuf., vol. 2, pp. 1-5, 2015.

[33] P. K. Dey, S. Hariharan, and B. T. Clegg, "Measuring the operational performance of intensive care units using the analytic hierarchy 
process approach," Int. J. Oper. Prod. Manag., vol. 26, no. 8, pp. 849-865, 2006)

[34] A. Cifalino and S. Baraldi, "Training programs and performance measurement Evidence from healthcare organisations," J. Hum. Resour. Costing Account., vol. 13, no. 4, pp. 294-315, 2007.

[35] C. Coulson-Thomas, "Transforming productivity and performance in healthcare and other public services : how training and development could make a more strategic contribution," Ind. Commer. Train., vol. 42, no. 5, pp. 251-259, 2010.

[36] R. J. M. M. Does, J. De Mast, and M. Schoonhoven, "Discussion of 'An Emerging Science of Improvement in Health Care ,"' Qual. Eng., vol. 27, pp. 35-40, 2015.

[37] B. R. Debata, B. Patnaik, S. S. Mahapatra, and K. Sree, "Interrelations of service quality and service loyalty dimensions in medical tourism," Benchmarking An Int. J., vol. 22, no. 1, pp. 18-55, 2015.

[38] A. Kumar, L. Ozdamar, and C. Peng Ng, "Procurement performance measurement system in the health care industry," Int. J. Health Care Qual. Assur., vol. 18, no. 2, pp. 152-166, 2005.

[39] A. Kumar, L. Ozdamar, and C. N. Zhang, "Supply chain redesign in the healthcare industry of Singapore," Supply Chain Manag. An Int. J., vol. 13, no. 2, pp. 95-103, 2008.

[40] D. Dobrzykowski, V. Saboori Deilami, P. Hong, and S.-C. Kim, "A structured analysis of operations and supply chain management research in healthcare (1982-2011)," Int. J. Prod. Econ., vol. 147, no. 2014, pp. 514-530, 2014.

[41] A. Y.-L. Chong and L. Zhou, "Demand chain management: Relationships between external antecedents, web-based integration and service innovation performance," Int. J. Prod. Econ., vol. 154, pp. 48-58, 2014.

[42] L. Chen and Y. Wang, "A conceptual framework for Taiwan' s hospital clinical performance indicators," J. Formos. Med. Assoc., vol. 114 , no. 5, pp. 381-383, 2015.

[43] I. G. Kwon, S. Kim, and D. G. Martin, "Healthcare supply chain management ; strategic areas for quality and financial improvement," Technol. Forecast. Soc. Chang., 2016.

[44] M. Falasca and J. F. Kros, "Success factors and performance outcomes of healthcare industrial vending systems: An empirical analysis," Technol. Forecast. Soc. Chang., 2016.
[45] C. F. Gomes and M. M. Yasin, "Assessing operational effectiveness in healthcare organizations : a systematic approach," Int. J. Health Care Qual. Assur., vol. 23, no. 2, pp. 127-140, 2010.

[46] J. de Vries and R. Huijsman, "Supply chain management in health services: an overview," Supply Chain Manag. An Int. J., vol. 16, no. 3, pp. 159-165, 2011.

[47] R. J. Al-Saa'da, Y. K. Abu Taleb, M. E. Al Abdallat, R. A. A. AlMahasneh, N. Awni Nimer, and G. a. Al-Weshah, "Supply Chain Management and Its Effect on Health Care Service Quality: Quantitative Evidence from Jordanian Private Hospitals," J. Manag. Strateg., vol. 4, no. 2, pp. 42-52, 2013.

[48] K. Katsaliaki, N. Mustafee, and S. Kumar, “A game-based approach towards facilitating decision making for perishable products: An example of blood supply chain," Expert Syst. Appl., vol. 41, no. 9, pp. 4043-4059, 2014

[49] S. Chakraborty, S. Bhattacharya, and D. D. Dobrzykowski, "Impact of Supply Chain Collaboration on Value Co-creation and Firm Performance: A Healthcare Service Sector Perspective," Procedia Econ. Financ., vol. 11, no. 14, pp. 676-694, 2014.

[50] S. A. Narayana, R. Kumar, and P. Vrat, "Journal of Purchasing \& Supply Management Managerial research on the pharmaceutical supply chain - A critical review and some insights for future directions," J. Purch. Supply Manag., vol. 20, no. 1, pp. 18-40, 2014.

[51] H. Aronsson, M. Abrahamsson, and K. Spens, "Developing lean and agile health care supply chains," Supply Chain Manag. An Int. J., vol. 16, no. 3, pp. 176-183, 2011.

[52] J. Parker and D. DeLay, "The future of the healthcare supply chain," Healthc. Financ. Manag., vol. 62, no. 4, pp. 66-69, 2008.

[53] H. K. Lee and Y. Fernando, "The antecedents and outcomes of the medical tourism supply chain," Tour. Manag., vol. 46, pp. 148-157, 2015

[54] A. A. Feil, D. M. De Quevedo, and D. Schreiber, "Selection and identification of the indicators for quickly measuring sustainability in micro and small furniture industries," Sustain. Prod. Consum., vol. 3, pp. 34-44, 2015. 